\title{
EXPERIMENTAL AND THEORETICAL INVESTIGATION OF MICRODISCHARGE PLASMA IN THE VORTEX GAS FLOW
}

\author{
V. Chernyak*, O. Kolomilets, O. Prysiazhna, O. Tsymbaliuk, \\ V. Iukhymenko, B. Portnyak, O. Fentisova, D. Nikulin \\ Taras Shevchenko National University of Kyiv, 64/13 Volodymyrska Street, 01601, Kyiv, Ukraine \\ * chernyak_v@ukr.net
}

\begin{abstract}
Experimental studies of the electrical parameters of the microdischarge and the plasma of the microdischarge in the vortex flow of $\mathrm{CO}_{2}$ as the plasma-forming gas was carried out. The kinetics of the formation of some components of microwave plasma was considered using ZDPlasKin computer code and Bolsig + at experimentally measured electric field strengths, pressure and gas temperature. The key reactions of microdischarge were determined. The Bolsig + code was used to determine the mean energy of electrons.
\end{abstract}

Keywords: atmospheric pressure plasma, $\mathrm{CO}_{2}$, microdischarge, plasma jet, plasma-chemistry.

\section{Introduction}

Plasma medicine is one of the newest areas of modern applied plasma chemistry. This area includes works related to a direct impact of atmospheric pressure low-temperature plasma on body tissues for various therapeutic effects. Moreover, this treatment must be non-destructive to the living organism.

Topical tasks for the plasma medicine are [1]: wounds sterilization, blood coagulation, treatment of skin diseases, selective programmed destruction of cancer cells, early diagnosis, etc. Some applications are already implemented with devices (kINPen MED [2], MicroPlasSter [3], PlasmaDerm [4], etc.).

Recent studies have shown the key role of reactive oxygen and nitrogen species (RONS) in medical applications. The effect of plasma-generated RONS on living tissues is described in [5]. The important fact is that plasma interacts with living organisms during plasma treatment. This forms a basis for the further studies in this direction, not only in the plasma medicine, but also in the agricultural applications.

Various plasma-forming gases are used in plasma generators: air, $\mathrm{He}, \mathrm{Ar}, \mathrm{CO}_{2}$, etc. It stands to reason that the kinetics of the processes that influence living tissues in all these cases will be different.

Earlier, microdischarge in air was investigated [6]. The system was used to treat mycelium and spores of mushrooms with further study of their growth [7].

In this work, we investigated the microdischarge in the $\mathrm{CO}_{2}$ vortex flow. The electrical parameters of the microdischarge and plasma emission spectra (200$1100 \mathrm{~nm}$ ) were experimentally investigated. Further study of plasma-chemical transformation processes was done by considering their kinetics using computer code ZDPlasKin. Bolsig + code was used to determine mean energy of electrons.

\section{Experimental setup}

The study was performed on an experimental setup presented in Figure 1. The microdischarge was axisymmetric plasma generator. Discharge was powered by DC power supply with negative high voltage potential. System had water cooling - 1 . Plasma-forming gas was supplied tangentially to a quartz chamber wall through a hole -2 . Microdischarge was burning between copper electrodes -3 . The high-voltage electrode was a wire with a thickness of approximately $4 \mathrm{~mm}$ with a rounded tapered tip with a radius of approximately $0.6 \mathrm{~mm}$. The external grounded electrode (anode) had the axial hole in the middle. Microdischarge jet was blown out through the axial hole with the diameter of $1 \mathrm{~mm}$. $\mathrm{CO}_{2}$ was used as a plasma-forming gas. The enlarged fragment on Figure 1 shows a schematic representation of the passage of gas through the current channel of the gas discharge.

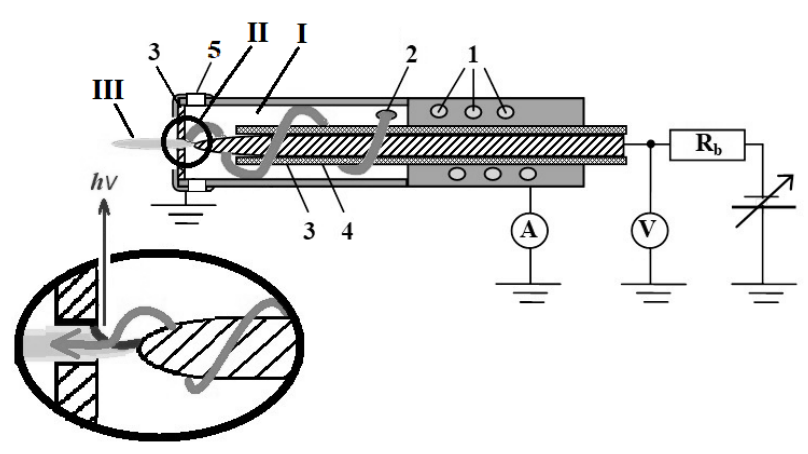

Figure 1. Experimental setup: 1 - holes of water cooling; 2-gas inlet; 3 - Cu electrodes; 4 -dielectric tube; 5 - quartz window.

Plasma-forming gas $\left(\mathrm{CO}_{2}\right)$ exits the reaction chamber through an axisymmetric opening, which passes perpendicularly to the discharge channel. The gas residence time in the discharge gap does not exceed $10^{-4} \mathrm{~s}$. As can be seen from Figure 1, there are three 


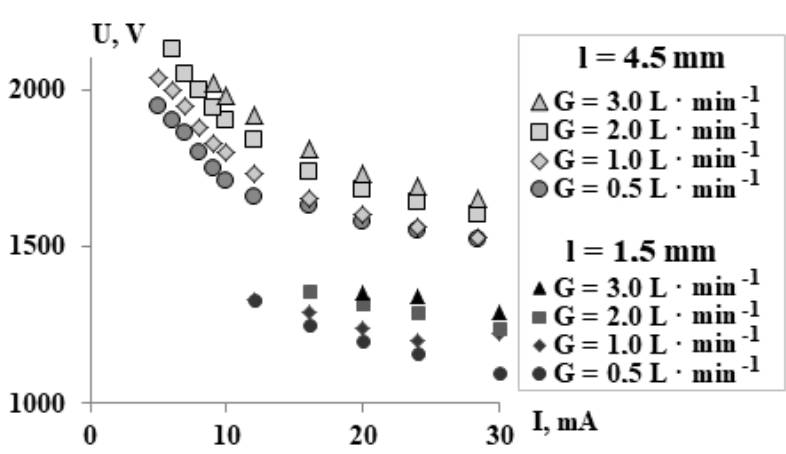

Figure 2. The current-voltage characteristics of microdischarge in the flow of $\mathrm{CO}_{2}$ plasma-forming gas $G=0.5 \div 3.0 \mathrm{~L} \mathrm{~min}^{-1}$ at the distances between electrodes $l=1.5 \mathrm{~mm}$ and $l=4.5 \mathrm{~mm}$.

main areas in which plasma-forming gas is located: I - inside the working chamber before entering the discharge current channel; II - the intersection area of the plasma-forming gas with the discharge area; III - plasma jet.

The parameters of the gas flow are selected for effective rotational gliding of the contacts of the current channel on the surface of the electrodes. The distance between the electrodes was adjusted within $0.5 \div 5.0 \mathrm{~cm}$ range with $0.5 \mathrm{~cm}$ step.

Based on the experimentally obtained data, the following parameters were used for computer simulation of microdischarge in atmospheric $\mathrm{CO}_{2}$ : electric field strength $E=1500 \mathrm{~V} \mathrm{~cm}^{-1}$, simulation time $t=\left(10^{-8} \div 1\right) \mathrm{s}$, time step $\Delta t=10^{-8} \mathrm{~s}$, temperature $T=1000 \mathrm{~K}[6]$.

\section{Results of experimental investigations}

Figure 2 presents the current-voltage characteristics of the microdischarge at different flows of plasmaforming gas $\mathrm{CO}_{2}\left(G=0.5 \div 3.0 \mathrm{~L} \mathrm{~min}^{-1}\right)$. The largest investigated value of the electrode distance was $l=$ $4.5 \mathrm{~mm}$, and the smallest was $l=1.5 \mathrm{~mm}$. It was observed that the increase of the distance between electrodes leads to more stable discharge burning for all studied gas flows. This is due to a significant increase in the breakdown voltage, which approaches the output voltage of the source. An increase in gas flow leads to an increase in voltage values at the same current values.

In contrast to the results obtained in the case of air [6], it was observed that in the case of using $\mathrm{CO}_{2}$ as a plasma-forming gas, the shortest electrode distance, at which the discharge was still burning, was $1.5 \mathrm{~mm}$. Moreover, in this case $(l=1.5 \mathrm{~mm})$ the study at different flows of plasma-forming gas $(G=0.5 \div$ $3.0 \mathrm{~L} \mathrm{~min}^{-1}$ ) showed unstable burning of the discharge at currents less than $12 \mathrm{~mA}(l=1.5 \mathrm{~mm}$ at Figure 2$)$.

Optical emission spectroscopy was performed near the surface of the grounded electrode. CCD-based Solar TII (S-150-2-3648 USB) spectrometer was used (op-

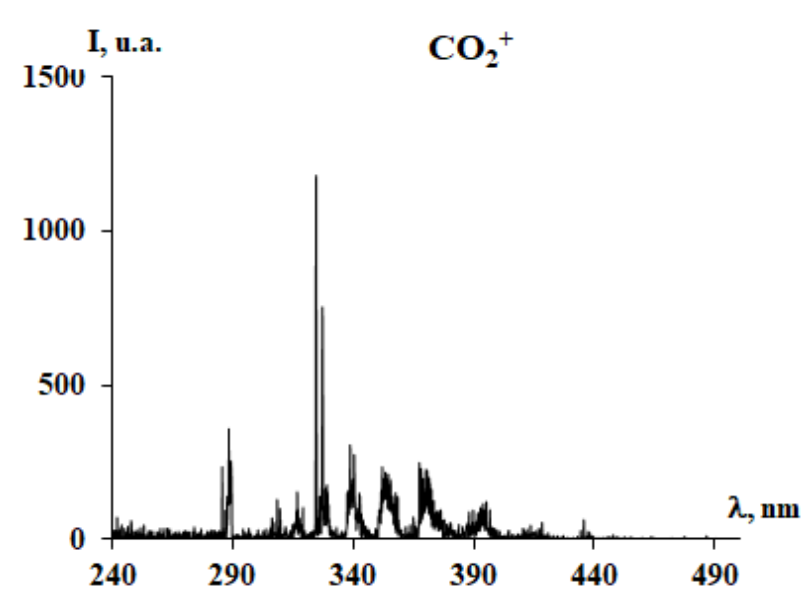

Figure 3. The experimental spectrum of microdischarge plasma recorded inside the discharge chamber in the wavelength range $\lambda=250 \div 500 \mathrm{~nm}\left(G=2 \mathrm{~L} \mathrm{~min}^{-1}\right.$; electrode distance $3 \mathrm{~mm} ; \mathrm{I}=30 \mathrm{~mA} ; \mathrm{U}=1200 \mathrm{~V})$.

erating in the wavelength range of $200 \div 1080 \mathrm{~nm}$ ). Figure 3 shows a typical experimental spectrum recorded under the following parameters: $G=2.0 \mathrm{~L} \mathrm{~min}^{-1}$; electrode distance $3 \mathrm{~mm} ; I=30 \mathrm{~mA} ; U=1200 \mathrm{~V}$. Data from [8] and program [9] were used in the analysis of spectroscopic data. Emission spectra of microdischarge plasma inside microdischarge system contain molecular bands of $\mathrm{CO}_{2}^{+}$. The electron excitation temperature of atomic oxygen was determined by the intensities of the atomic $O$ lines at $777.6 \mathrm{~nm}, 844.9 \mathrm{~nm}$, $927.2 \mathrm{~nm}$. For microdischarge with an electrode distance of $l=2 \mathrm{~mm}$, a change in current $I=20 \div 30 \mathrm{~mA}$ led to an increase in temperature by $1000 \mathrm{~K}$ (Figure 4). An increase in the flow of plasma-forming gas $G=1 \div 3 \mathrm{~L} \mathrm{~min}^{-1}$ led to an increase in temperature by $1300 \mathrm{~K}$. For microdischarge with the electrode distance of $l=3 \mathrm{~mm}$ a similar trend was not observed.

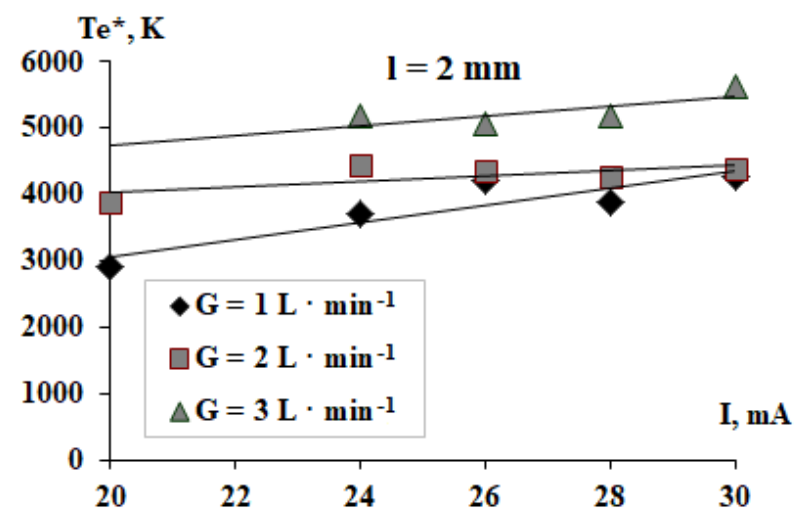

Figure 4. The electron excitation temperature of $O(777.6 \mathrm{~nm}, 844.9 \mathrm{~nm}, 927.2 \mathrm{~nm})$ for $l=2.0 \mathrm{~mm}$, $I=20 \div 30 \mathrm{~mA}$ and $\mathrm{G}=1 \div 3 \mathrm{~L} \mathrm{~min}^{-1}$.

Current-voltage characteristics were used to build the dependencies of discharge voltage on electrode distance at fixed current value. From these graphs the 
electric field for $\Delta l=1 \mathrm{~mm}$ was found (Figure 5 ). The electric field was determined using the tangent of the slope angle of the linear sections of the dependence of the discharge voltage drop on the distance between the electrodes, since in these experiments the region of the cathode voltage drop is much smaller than the distance between the electrodes. It can be seen that an increase in the plasma-forming gas flow leads to an increase in the discharge voltage. The obtained graphs are not linear: each dependency has three sections. The first and third sections are growing, and the second one is sloping. In the linear sections of Figure 5, estimates of the reduced electric field were made. The results are shown in table 1 .

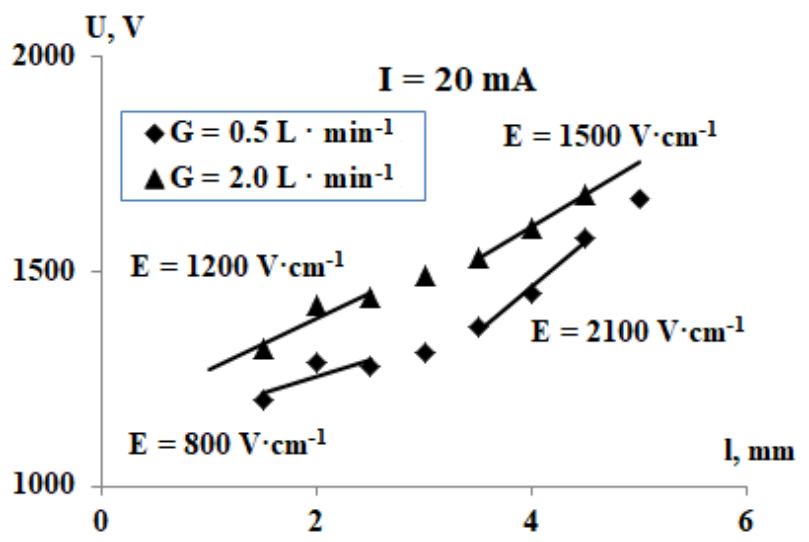

Figure 5. Reduced electric field of microdischarge in $\mathrm{CO}_{2}$ jet at different distances between electrodes.

\begin{tabular}{ccc}
\hline & \multicolumn{2}{c}{$\Delta l$} \\
\cline { 2 - 3 }$G \mathbf{~ L ~ m i n}^{-1}$ & $\mathbf{1 . 5} \div \mathbf{2 . 5} \mathbf{~ m m}$ & $\mathbf{3 . 5} \div \mathbf{4 . 5} \mathbf{~ m m}$ \\
\hline 0.5 & $11 \mathrm{Td}$ & $29 \mathrm{Td}$ \\
2.0 & $16 \mathrm{Td}$ & $21 \mathrm{Td}$ \\
\hline \hline
\end{tabular}

Table 1. The Townsend values of reduced electric field of microdischarge in $\mathrm{CO}_{2}$ jet at different distances between electrodes.

The study of kinetics of processes in microdischarge plasma was performed using numerical simulations. The computer code ZDPlasKin allows us to simulate the time evolution of plasma composition under the conditions of constant electric field, fixed values for temperature of plasma-forming gas, pressure and given gas composition. During the calculation, a database containing more than 1500 conversion processes was used. The Bolsig + code was used to model the kinetics of processes involving the participation of electrons under the conditions of constant electric field, temperature of plasma-forming gas, pressure and plasma-forming gas composition. More than 100 components were taken into account in the calculation, as well as transformations involving vibrationally excited molecules. The rate constants of these processes were entered into the database according to the FridmanMacheret theory [1]. Calculated reduced electric field in the range of was used in Bolsig + to determine the mean electron energy.

Figure 6 shows the concentration dependences for the main neutral components of microdischarge plasma in the $\mathrm{CO}_{2}$ flow: excited atoms $\mathrm{O}-\mathrm{O}\left({ }^{3} \mathrm{P}\right), \mathrm{O}(1 \mathrm{D})-\mathrm{O}\left({ }^{1} \mathrm{D}_{2} 2 \mathrm{p}^{4}\right)$; vibrationally excited molecules in the ground electron state $\mathrm{O}_{2}-\mathrm{O}_{2}(\mathrm{X}, \mathrm{v}=0) ; \mathrm{O}_{2}(\mathrm{~V} 1)-\mathrm{O}_{2}(\mathrm{X}, \mathrm{v}=1) ; \mathrm{CO}-$ $\mathrm{CO}(\mathrm{X}, \mathrm{v}=0) ; \mathrm{CO}_{2}-\mathrm{CO}_{2}(\mathrm{X}, \mathrm{v}=0) ; \mathrm{CO}(\mathrm{V} 1)-$ $\mathrm{CO}(\mathrm{X}, \mathrm{v}=1) ; \mathrm{CO}_{2}(\mathrm{~V} 1)-\mathrm{CO}_{2}(\mathrm{X}, \mathrm{v}=1) ; \mathrm{CO}_{2}(\mathrm{~V} 4)-$ $\mathrm{CO}_{2}(\mathrm{X}, \mathrm{v}=4)$ and molecules in electronically excited states $\mathrm{O}_{2}(\mathrm{~B} 1)-\mathrm{O}_{2}\left(\mathrm{~b}^{1} \Sigma_{g}^{+}\right)$.

It should be mentioned that oxygen reactive particles play a key role in plasma medicine. Therefore, we will next consider the reactions responsible for the atomic oxygen formation in the microdischarge.

In Figure 6 it is possible to observe the evolution of $\mathrm{CO}_{2}$ and $\mathrm{CO}$ components over time during the conversion of the plasma-forming $\mathrm{CO}_{2}$ gas with the participation of an oxidant.

Kinetics modeling of microdischarge plasma processes in the $\mathrm{CO}_{2}$ jet were carried out for gas temperatures $T=500 \mathrm{~K}$ and $T=1000 \mathrm{~K}$ (Figure 6 ). These temperatures were chosen based on the values obtained from the experimental spectra of microplasma.

The dependencies of the simulated concentrations of the main components of the microdischarge plasma in the $\mathrm{CO}_{2}$ jet from the time of plasma gas exposure are shown in Figure 6 for a) $T=500 \mathrm{~K}$ and b) $T=1000 \mathrm{~K}$. These results show that in the plasma of $\mathrm{CO}_{2}$ there are more excited atomic components than excited molecular components. This indicates the significant activity of such plasma and its promising use in plasma medicine. The concentration of $\mathrm{O}$ atoms using $\mathrm{CO}_{2}$ as plasma-forming gas is substantially higher than when using humid air [10].

The dependencies of the concentrations of excited atoms $(\mathrm{O})$ and molecules $\left(\mathrm{O}_{2}, \mathrm{CO}, \mathrm{CO}_{2}\right)$ on the excitation energy of the corresponding states are shown in Figure 7. Obtained dependencies indicate the equilibrium character of the populations of electron levels of oxygen atoms and the vibration levels of $\mathrm{O}_{2}, \mathrm{CO}_{2}$, and $\mathrm{CO}$ molecules at temperatures a) $T=500 \mathrm{~K}$ and b) $T=1000 \mathrm{~K}$. The electron excitation temperature, which was determined from the experimental spectra Figure 4, matched with the calculated electron excitation temperature $\left(T_{\mathrm{e}}^{*}(\mathrm{O})=0.45 \mathrm{eV}=5200 \mathrm{~K}\right)$.

It can be seen from Figure 7 that increase of the gas temperature leads to a decrease in the vibrational temperature of $\mathrm{O}_{2}$ molecules and electronic excitation temperature of oxygen atoms and to an increase in the electron excitation temperature of $\mathrm{O}_{2}$ and vibrational temperatures of $\mathrm{CO}_{2}$ and $\mathrm{CO}$ molecules respectively to the Figure 6. 


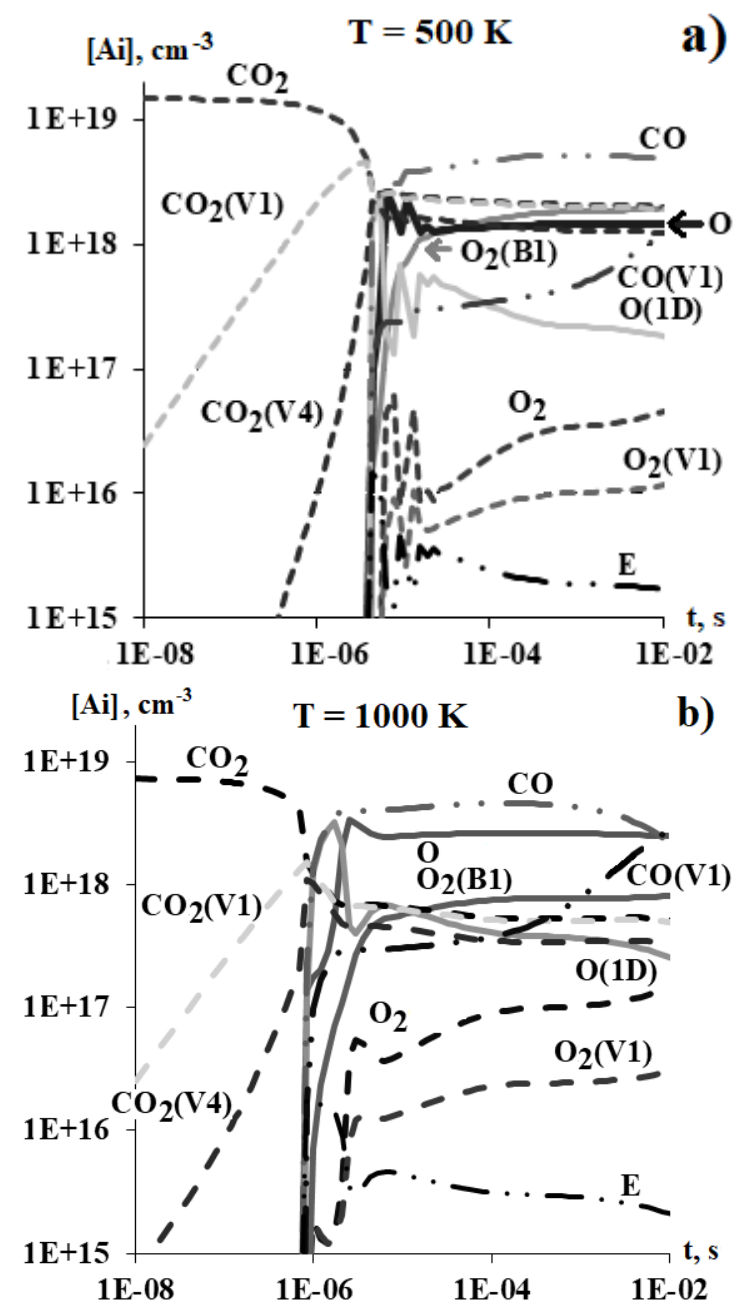

Figure 6. Dependence of modeled concentrations of microdischarge plasma components in $\mathrm{CO}_{2}$ flow on time of plasma gas exposure for a) $T=500 \mathrm{~K}$ and b) $T=1000 \mathrm{~K}$.

\section{Conclusions}

The electric field of the microdischarge in the $\mathrm{CO}_{2}$ flow at smaller erelectrode distances $(l=1.5 \div 2.5 \mathrm{~mm})$ has smaller values compared to the values at larger electrode distance $(l=3.5 \div 4.5 \mathrm{~mm})$. (The opposite ratios were obtained for plasma-forming gas - Air).

Electron energy distribution functions are not Maxwell distributions.

The simulation results indicate an equilibrium population distribution of the electron levels of the oxygen atoms and the vibration levels of molecules of $\mathrm{O}_{2}$, $\mathrm{CO}_{2}$, and $\mathrm{CO}$ for $t \sim 10^{-4}$ sec.

The calculated electron excitation temperature of atomic oxygen corresponds to the temperature determined from the experimentally obtained emission spectra.

In the $\mathrm{CO}_{2}$ plasma the concentration of oxygen atoms is significantly higher than the concentration of oxygen molecules. This indicates the significant activity of such plasma and its promising use in plasma medicine.

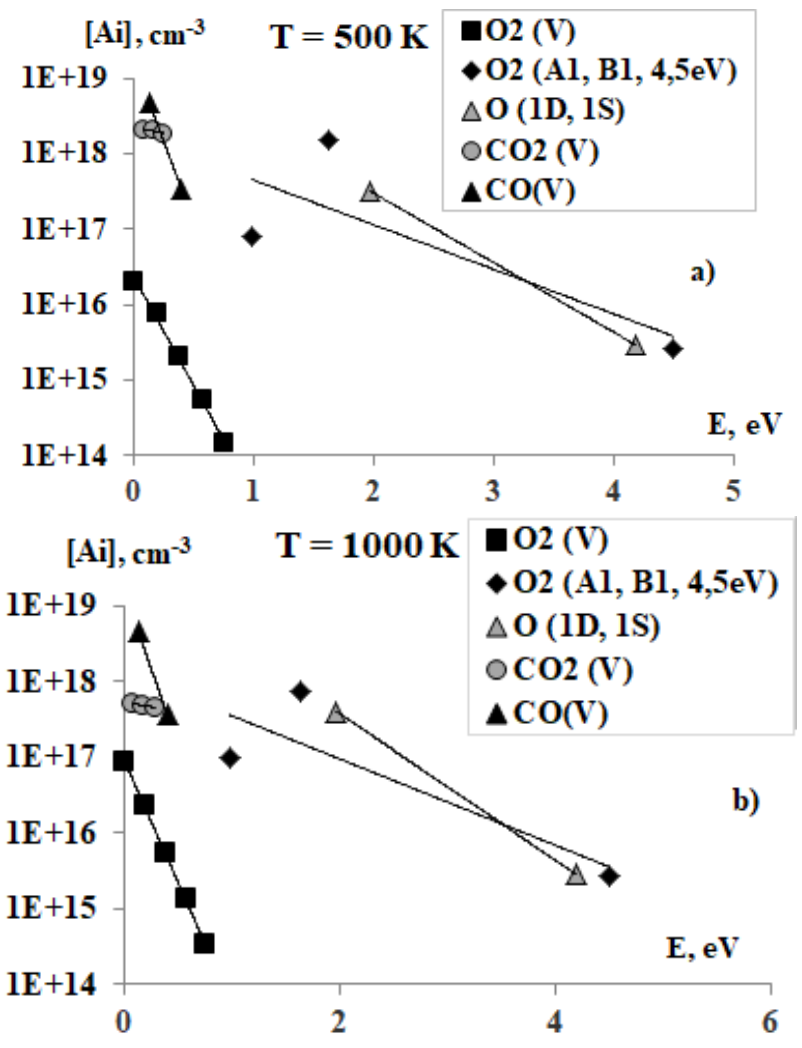

Figure 7. Dependence of modeled concentrations of microdischarge plasma components in $\mathrm{CO}_{2}$ flow on excitation energy of corresponding state for $t \sim 10^{-4} \mathrm{sec}$ a) $\mathrm{T}=500 \mathrm{~K}$ and b) $\mathrm{T}=1000 \mathrm{~K}$.

\section{Acknowledgements}

This work was partially supported by the Ministry of Education and Science of Ukraine and Taras Shevchenko National University of Kyiv.

\section{References}

[1] A. Fridman. Plasma Chemistry. Cambridge University Press, Cambridge, 2008.

[2] R. Schönebeck. kINPen MED ${ }^{\circledR}$. In Comprehensive Clinical Plasma Medicine, chapter 32. Springer, Cham, 2018.

[3] A. Schmidt and S. Bekeschus. Redox for Repair: Cold Physical Plasmas and Nrf2 Signaling Promoting Wound Healing. Antioxidants, 7(10):146, 2018.

doi:10.3390/antiox7100146.

[4] D. Wandke. PlasmaDerm ${ }^{\circledR}$ - Based on di_CAP Technology. In Comprehensive Clinical Plasma Medicine, chapter 33. Springer, Cham, 2018.

[5] C. Duchesne, N. Frescaline, J.-J. Lataillade, and A. Rousseau. Comparative study between direct and indirect treatment with cold atmospheric plasma on in vitro and in vivo models of wound healing. Plasma Medicine, 8(4), 2018.

doi:10.1615/PlasmaMed. 2019028659. 
[6] V. Chernyak, O. Kolomiiets, V. Iukhymenko, O. Tsymbaliuk, V. Khomiak, and D. Chernysh. Properties of microdischarge plasma in the vortex air flow. Problems of Atomic Science and Technology, (6), 2018 .

[7] Y. Veremii, I. Andriiash, N. Tsvyd, V. Chernyak, M. Sukhomlyn, and E. Martysh. Influence of cold atmospheric plasma of microdischarge on fungal mycelium and spores growing. Problems of Atomic Science and Technology, (1), 2019.

[8] R. W. B. Pearse and A. G. Gaydon. The identification of molecular spectra. London: Chapman and Hall; New York: Wiley, 1976.
[9] Z. Navrátil, D. Trunec, R. Šmíd, and L. Lazar. A software for optical emission spectroscopy-problem formulation and application to plasma diagnostics. Czechoslovak Journal of Physics, 56(2):B944-B951, 2006. doi:10.1007/s10582-006-0308-y.

[10] V. Chernyak, O. Tsymbaliuk, D. Levko, E. Martysh, V. Iukhymenko, O. Kolomiets, V. Khomijak,

D. Chernysh, and O. Fentisova. Kinetics of processes in air plasma discharges at atmospheric pressure in the transverse flow of gas. Problems of Atomic Science and Technology, (1), 2019. 